\title{
A New Ontological View of the Quantum Measurement Problem
}

\author{
Xiaolei Zhang \\ Code 7218 \\ US Naval Research Laboratory \\ 4555 Overlook Ave. SW \\ Washington, DC 20375, USA \\ e-mail: xzhang@nrl.navy.mil
}

Naval Research Lab Memoradum Report

NRL/MR/7218-05-8883

July 3, 2021 


\section{Abstract}

A new ontological view of the quantum measurement processes is given, which has bearings on many broader issues in the foundations of quantum mechanics as well. In this scenario a quantum measurement is a non-equilibrium phase transition in a "resonant cavity" formed by the entire physical universe including all of its material and energy content. A quantum measurement involves the energy and matter exchange among not only the system being measured and the measuring apparatus but also the global environment of the universe resonant cavity, which together constrain the nature of the phase transition.

Strict realism, including strict energy and angular momentum conservation, is recovered in this view of the quantum measurement process beyond the limit set by the uncertainty relations, which are themselves derived from the exact commutation relations for quantum conjugate variables. Both the amplitude and the phase of the quantum mechanical wavefunction acquire substantial meanings in the new ontology, and the probabilistic element is removed from the foundations of quantum mechanics, its apparent presence in the quantum measurement being solely a result of the sensitive dependence on initial/boundary conditions of the phase transitions of a many degree-offreedom system which is effectively the whole universe. Vacuum fluctuations are viewed as the "left over" fluctuations after forming the whole numbers of nonequilibrium resonant modes in the universe cavity.

This new view on the quantum processes helps to clarify many puzzles in the foundations of quantum mechanics, such as wave-particle duality, Schrodinger's Cat paradox, first and higher order coherence of photons and atoms, virtual particles, the existence of commutation relations and quantized behavior, etc. It naturally explains also the appearance of a hierarchy of structures in the physical universe as the result of successive spontaneous phase transitions under natural boundary conditions, whose occurrence does not need the presence of conscious observers to "collapse the wavefunction". Implications of the new view on the currently successful approaches in quantum field theories, such as the renormalization procedure and Feynman diagrammatic approach, are also discussed. 


\section{Introduction}

The advent of quantum mechanics eight decades ago and the accompanying Copenhagen interpretation marked the beginning of a new phase of physics research in the microscopic domain, whereupon ontological realism was generally abandoned for computational proficiency. Despite the successful demonstration of statistical correspondence between quantum theory and experiments (Wheeler \& Zurek eds. 1983 and the references therein), as well as the even more spectacular demonstration of the quantitative agreement between the renormalized quantum electrodynamics calculations and experimental results on fundamental quantities such as Lamb shift and the anomalous magnetic moment of the electron (see, e.g., Nambu 1949 and the references therein), a cloud of unease lingers over the loss of a concrete and realistic image of the physical world, compared to that of the classical world which we were used to.

Central to this unease is the quantum measurement problem and the associated probabilistic interpretation of the quantum mechanical wavefunction which predicts in the statistical sense the outcome of any quantum measurement. As eloquently summarized in a recent article by DeWiit (2005): "If one accepts the view that formalism and reality are isomorphic, then in the quantum theory one is obliged to accept a stupendous number of simultaneous realities, namely, all the possible outcomes of quantum measurements as well as all the possible 'classical' worlds that emerge spontaneously from the wavefunction of the universe through the phenomenon of decoherence". Even without being a whole-hearted convert to the above many-world view, one is still left wondering about the underlying implications of the probability interpretation.

As is well-known in the classical world, the statistical and probabilistic phenomenology of a physical process does not necessarily imply an underlying propabilistic ontology. Likewise, the propabilistic outcome of the quantum measurement does not necessarily warrant a "many world" ontology, especially if this implies giving up energy conservation which is one of the cornerstones of the physical sciences. 
The unease results also from the need to artificially draw a line between the behaviors of the quantum and the classical world. Since in the physical universe the large and the small scale phenomena are often intermingled, and even in quantum measurement of microscopic processes the apparatus involved are of macroscopic dimensions, this lack of a clear dividing line between the two worlds necessarily leads to paradoxes such as "Schrodinger's Cat paradox" (Schrodinger 1935), and the seemingly ridiculous need for a conscious observer to "collapse the wavefunction" in order for the measurement result to settle onto one of the many possibilities dictated by quantum calculations (Von Neumann 1932).

Part of the goals of the current paper is to restore realism to the ontological foundations of quantum mechanics, and to bridge the connection between the quantum and the classical worlds. This work goes beyond the orthodox Copenhagen Interpretation, and discredits the uncertainty relation as a fundamental law of physics, and shows rather that the commutation relations, from which the uncertainty relations can be derived, describe quantitatively the behavior of the quantized resonant modes in the universe cavity. In the new ontology the concepts of nonequilibrium phase transition as well as the generalized Mach's principle underlie the explanation of all quantized phenomena. A coherent picture can be arrived at which synthesizes also the results from the past decades on S-matrix theories, quantum electrodynamics and gauge field theories, and offers a clearer explanation of phenomena both in the quantum as well as in the classical world.

The organization of the paper is as follows: Section 2 outlines the essential features of the quantum measurement process, and ascertain that the so-called "quantum wavefunction collapse" is real and is prevalent in quantum measurements. It also review several of the previously proposed theories on quantum measurement. Section 3, which forms the core of this paper, presents the new ontological view of the quantum measurement process, gives its motivation and main assumptions, as well as the implications of the new ontology on resolving and coherently interpreting many issues in the foundations and frontiers of quantum mechanics. The remaining unanswered questions which come into sharper focus under this new picture will also be outlined. Finally, section 4 summarizes the conclusions of this work. 


\section{The Quantum Measurement Problem}

\subsection{Distinguishing Features of Quantum Wavefunction Collapse}

Whereas a classical measurement usually leads to a definitive result given by deterministic classical calculations, the result of a quantum measurement in general can only be predicted in a probabilistic sense. The quantum mechanical wavefunction is the most quantitative result from a quantum calculation, and one can hope for a definitive answer only if the measurement is towards an eigenvalue of a system already settled onto its corresponding eigenstate. If this is not so, the underlying system is assumed to subsequently "collapse" onto an eigenstate of the measurement operator with the probability for the choice of state given by the absolute square of the wavefunction.

First of all, we must convince ourselves that there is sufficient evidence to indicate that such "wavefunction collapse" does happen during quantum measurement, and that a quantum system under measurement behaves in essentially different ways from classical systems.

Most of us are familiar with the position-momentum uncertainty relation. The quantum mechanical wavefunction of a particle in momentum eigenstate $\mathbf{p}_{\mathbf{0}}$ is expressed as the following plane wave

$$
u_{\mathbf{p}_{\mathbf{0}}}(\mathbf{r})=\frac{1}{\sqrt{(2 \pi \hbar)^{3}}} \exp ^{\frac{i}{\hbar} \mathbf{p}_{\mathbf{0}} \cdot \mathbf{r}}
$$

so a momentum measurement will lead to a definite result $\mathbf{p}_{\mathbf{0}}$. However, if this momentum measurement is followed by a positional measurement, the resulting position can take any value with equal probability, as indicated by the constant amplitude of (1) with respect to the values of $\mathbf{r}$. But once the position measurement is performed and obtained a specific value $\mathbf{r}_{\mathbf{0}}$, the wavefunction "jumps" to the new form of

$$
u_{\mathbf{r}_{\mathbf{0}}}(\mathbf{r})=\delta\left(\mathbf{r}-\mathbf{r}_{\mathbf{0}}\right),
$$

the subsequent momentum measurement once again becomes uncertain, and every value of the momentum is equally likely to be obtained. The "particle" 
thus alternates between possessing a position-eigenstate wavefunction and a momentum-eigenstate wavefunction while undergoing alternating position and momentum measurements.

Other evidence of the wavefunction collapse are found in the experiments described in Chiao et al. (1994 and the references therein) using photons, and Berman ed. (1997 and the references therein) using atoms. In such experiments the photons and atoms are spread out, i.e., in mostly momentum eigenstate, just prior to detection, and are localized at the instant of detection. This feature is confirmed in many of the "delayed choice" type of experiments (Wheeler 1978; Alley et al. 1987; Hellmuth et al. 1987; Briegel et a. 1997).

Other examples which display the distinctive characteristics of the quantum measurement process include the Aspect et al. experiment (Aspect 1976; Aspect, Grangier \& Roger 1981) to test Bell's inequality (Bell 1965) and quantum entanglement, which revealed the existence of the action-at-adistance type of nonlocal communication during the quantum measurement process. Special relativity and Lorentz invariance appear to be violated in both the entanglement type experiments and in the broader quantum measurements which involve an instantaneous wavefunction collapse.

Another characteristic feature of the wavefunction collapse is that energy appears not to be conserved, at least not among the measuring apparatus and the system being measured. For example, the detection of the position of a particle makes its momentum, and thus energy, uncertain. Since this uncertain energy could in principle be infinite, the question comes as to where the additional energy has arrived from. Even for resonant interaction, it was known that "... transitions within short times occur not only between states which satisfy the condition $E+\epsilon=E^{\prime}+\epsilon^{\prime}$ ( $E$ and $E^{\prime}$ being the energy of the system before and after the transition, $\epsilon$ and $\epsilon^{\prime}$ that of the apparatus). These states are given preference by resonance only after a long time. In practice, after a time $\Delta t$, only transitions by which $\left|E+\epsilon-E^{\prime}-\epsilon^{\prime}\right|<=h_{\text {bar }} / \Delta t$ are of importance (Landau and Peierls 1931)". The apparent energy nonconservation is also present in processes involving virtual particles. 


\subsection{Existing Quantum Measurement Theories}

The many aspects of the quantum measurement processes and the various versions of quantum measurement theories can be found in several books and references on the subject. See, e.g., Wheeler \& Zurek (1983), Braginsky \& Khalili (1992), Namiki, Pascazio, \& Nakazato (1997) and the references therein.

\subsubsection{The Copenhagen Interpretation}

The orthodox Copenhagen Interpretation (see, e.g. Bohr 1928) for quantum processes emerged at the wake of Born's propabilistic interpretation of the quantum mechanical wavefunction (Born 1926) and Heisenberg's uncertainty principle (Heisenberg 1927), and incorporates also Bohr's own Principle of Complementarity. It emphasizes the need for classical description of quantum measurement results, but requires a clear (but somewhat arbitrary) distinction between the quantumness of the system being measured, and the classicality of the measuring instrument. It introduces formally the element of "wavefunction collapse" into the description of quantum measurement, but gives no explanation of the nature or cause of the collapse. Neither does it allow any substantial interpretation of the wavefunction itself. It is at best only a compromise for the lack of a true ontological theory. Its success lies solely in the phenomenological description of the quantum world and in predicting the quantum measurement results in a probabilistic sense. The Copenhagen Interpretation started the trend of "shut-up and calculate" practice which still dominates the scene of quantum research eight decades after its invention.

\subsection{2 "Many World" Interpretation}

The "many world" theory of Everett III (1957) and DeWitt (1970) did away with the quantum jumps of the standard Copenhagen picture, and proposes instead that the quantum world is governed only by the linear and continuous evolution of Schrodinger type. The many possibilities of reality offered by 
the Schrodinger's equation are all realized, and they branch away from one another at every instant. We are only aware of one of the possibilities because we reside only on one of the branches.

This theory itself obviously is not falsifiable, and does not constitute a bona fide scientific theory in the sense of Popper (1935). It also conflicts with the realist tradition, and does not offer an explanation of where the energy of all the "branching aways" comes from. Neither does it help understanding

quantum features such as the wave-particle duality in a double-slit type of experiment.

\subsection{3 "Decoherence" Theories}

The modern decoherence theories (Wigner 1963; Zurek 2002 and the references therein) originated from J. Von Neumann's quantum measurement theory (Von Neumann 1932). A common theme in these theories is that the measurement process is viewed as a multi-step process, with the initial interaction of the system being measured and the measuring apparatus bringing about a correlation between the two, and their states becoming entangled. Subsequently, the density matrix describing such a entangled pure state loses its off-diagonal elements (either spontaneously as in the case of von Newmann's version, or through interaction with the environment as in the Zurek's version), a process which is termed "decoherence" or "reduction of wavefunction", and the density matrix of the measurement process thus becomes that of a mixture state instead of a pure state. Following this step, one usually has to resort to the intervention of conscious observers to finally pick from one of the many classical probabilities indicated by the nonvanishing diagonal elements of the "decohered" density matrix.

The decoherence theory gives no true cause of the loss of the off-diagonal elements (coupling with a thermal dissipative environment does not describe the nature of all quantum measurement processes, not even the most classical ones such as successive position/momentum measurements). The further need for conscious observers to intervene to decide on one of the classical possibilities is also in conflict with what we observe in the natural world where 
processes happen without the intervention of human intelligence. There is also no explanation in this picture of what the rest of the potential classical outcomes mean and what happen to them when a conscious being picks out one among the many.

As we will see below, in the quantum to classical transition, the real issue is not the vanishing of the off-diagonal interference term in the density matrix (i.e. the so-called decoherence), but rather the discontinuous evolution of the wavefunction at the moment of measurement, described as a non-equilibrium phase transition.

\section{The New Ontology}

In the classical world, all quantized phenomena we know of are related to resonances in a particular type of cavity, either closed (as in the equilibrium resonance phenomena) or open (as in the nonequilibrium resonance phenomena). In particular, the nonequilibrium resonance phenomena are closely related to the nonequilibrium phase transitions, and the structures so formed in the underlying open, far-from-equilibrium systems are termed "dissipative structures" (Glansdorf \& Prigogine 1971; Nicolis \& Prigogine 1977). Some classical examples of dissipative structures are the turbulent convection cells in atmosphere circulation (Benard's instability, see, e.g. Koschmieder 1993 and the references therein), and the spiral structure in galaxies (Zhang $1996,1998,1999,2003,2004)$. A close analogy to such a nonequilibrium phase transition is the three-dimensional stereo image formation by the human eyes and brain when observing two separate stereo images: The formation of the three-dimensional image in the brain is obviously not a simple linear and sequential optical interaction, but rather pattern formation through spontaneous symmetry breaking. Once formed, the new pattern is stable against small perturbations. Dissipative structures are in dynamical equilibrium their maintenance depends on a constant flux of energy and entropy through the system, and the exchange with their environment.

The universe is an open and evolving system. If we insist on a realist interpretation of the physical processes, as well as on a continuous classical 
and quantum interface, we have to seek the origin of the quantized behavior in resonance phenomena. Since physical constants such as Planck's constant are universal, and different types of interaction processes in vastly different environments give the same elementary particle properties, such resonant behavior are expected to originate from the entire matter and energy content of the universe. Such a view is closely related to Mach's principle for explaining the origin of mass and inertia (see, e.g., Barbour \& Pfister 1995 and the references therein), and forms the starting point of the new ontological view of quantum mechanics we are presenting in this paper.

In what follows in this section, we will first list the central postulates of this ontology, and then provide further justifications through the comparison with empirical evidence, the work of the past few decades in quantum field theories, and some earlier speculations of the pioneering workers.

\subsection{Fundamental Postulates}

- It is assumed that a generalized form of "Mach's Principle" governs the operation of the physical universe, and that the local properties of matter, including the values of fundamental constants and the forms of physical laws, are determined by the global distribution of all the matter and energy content in the universe.

- The physical universe is organized into hierarchies (as reflected in part in the spontaneous breaking of gauge symmetry in the generation of physical laws). The quantum/classical hierarchy is divided through spontaneous nonequilibrium phase transitions which form the macroscopic structures. These macroscopic structures are capable of resisting the diffusion/smearing tendency of pure quantum states governed by Schrodinger-type wave equations and can thus remain quasi-stable.

- The quantized nature of fundamental processes originates from nonequilibrium phase transitions in the universe resonant cavity. The usual quantization procedure by enforcing the commutation relations is equivalent to establishing modal closure relations in the universe cavity. Uncertainty relations are only the phenomenological derivatives of the 
corresponding commutation relations and they themselves have no independent fundamental significance.

- A quantum measurement process is equivalent to setting up an appropriate boundary condition so that a phase transition in the joint system of object, measuring instrument and the rest of the universe is induced.

- The quantum mechanical wavefunction describes the substantial distribution of the underlying matter of specific modal type. Its absolute square gives the probability for obtaining a particular configuration in the measurement phase transition, and its phase encodes the influence of the environment which determines its subsequent evolution. The probabilistic element is thus removed from the ontology of quantum mechanics.

- The evolution of physical systems in the universe is described by successive stages of continuous wavefunction evolution and discontinuous phase transitions. The universe as a whole is neither described by a single autonomous wavefunction, nor is it a statistical mixture. It is organized rather as a hierarchy of nearly independently-evolving subsystems, with the division of the hierarchies accomplished by successive stages of phase transitions, though the interaction and exchanges among the hierarchies also happen during the same phase transitions.

- In this picture the vacuum fluctuations are the "residuals" of the making of the "whole" numbers of non-equilibrium quasi-stationary modes in the open, nonequilibrium universe cavity.

\subsection{Implications on the Foundations of Quantum Me- chanics}

The new ontology has immediate implications for obtaining a coherent picture of the observed quantum phenomena and for bridging the connection with the classical world. The effectiveness of these interpretations also serves as empirical support for the validity of our fundamental postulates. 


\subsubsection{The Meaning of the Quantum Mechanical Wavefunction}

When Schrodinger first derived his wave mechanics formulation, he envisioned the wavefunction as representing the electron density, and a point electron as a superpositional wave packet (Schrodinger 1926a). This view was criticized (notably by Lorentz, see, e.g. Moore 1989, p. 216) and Schrodinger himself subsequently abandoned it. There are mainly two problems with this view. First of all, a wavepacket initially localized in space is found to disperse with time when the time-dependent Schrodinger equation is solved, especially if the size of the wavepacket is not much larger than the de Broglie wavelength. Secondly, as Schrodinger has commented: "The $\Psi$ function itself cannot and may not be interpreted directly in terms of three-dimensional space - however much the one electron problem tends to mislead us on this point - because it is in general a function in (3n-dimensional) configuration space, not real space" (Schrodinger 1926).

The commonly accepted interpretation of the wavefunction in the nonrelativistic quantum mechanics is the probabilistic interpretation first suggested by Max Born (1926). Quantum mechanics yields only probabilities for the outcomes of atomic events, but the probabilities themselves evolve in a precise, deterministic fashion in accordance with the Schrodinger equation. The wavefunction is thus stripped of any substantial meaning and is considered only as a probability wave. In quantum field theories, however, a certain realist element can be discerned within the overall probabilistic framework. This is because quantum field theories already incorporated many of the modal features implicitly.

In our current ontology, the realist view of the wavefunction is fully recovered. The probability wave is now a physical entity, the chance factor only enters later, at the moment of measurement. The fact that the wavefunction exists in the 3n-dimensional configuration space no longer poses a problem, since this signifies only the interchange and interrelation of the different parcels of the modal content among a multi-particle state. Thus, the coordinates of choice for the multi-particle wavefunction could be any of the abstract canonical coordinates of Hamilton's mechanics, since these can equally effectively express modal characteristics. Furthermore, the dispersion 
of the wavepacket is a natural feature of the superpositional state for a localized "particle". Schrodinger's earlier confusion lies in regarding the localized particle as a pure resonant state, and he struggled with preserving the localization of the wavepacket. In actuality the momentum eigenstate, which is spread out in space, is often a more "natural" state for a particle resonance, as we will comment further below, thus an isolated positional state tends to diffuse towards it.

The probability factor in the result of quantum measurement is due to the intrinsic sensitive dependence on initial/boundary conditions of the phase transitions happening in a many degree-of-freedom system. Just as its classical counterpart, the underlying cause of this probability factor is deterministic though generally not "determinable" due to the difficulty of tracking down all the causes in an effective infinite degree-of-freedom system which contain the evolving wavefunctions and quasi-steady states of all the matter in the universe.

Whereas the amplitude of the wavefunction is correlated with the modal density in the configuration space, and can thus lead to the probability of a particular measurement outcome as in the Born's interpretation, the phase of the wavefunction is related to the interaction fields in the gauge field formulation. As C.N Yang puts it, "all fundamental forces are phase fields (Yang 1987)".

The Aharonov-Bohm effect (Aharonov \& Bohm 1959) which shows the influence to the phase in the region where the nominal field strength is absent demonstrate that it is the relative change in the phase of the wavefunction of the electrons produced by the potential that is physically observable. The change is not produced by any local interaction of the potential with the electrons. Rather, it is dictated by a certain global property of the potential specified by a gauge function.

In the new ontology the quantum mechanical wavefunction reflects the self-organization of the matter contents in the universe into modal form. The wavefunction of a quantum observable usually spreads throughout in the infinite space, and the interaction between the different observables/modes are global in general, which is the reason that quantum mechanics is formulated 
in the Hilbert space, a natural domain to describe abstract global modal relations. A quantum wavefunction continuously evolves and can be superposed as in the interference effect. Measurement phase transition introduces nonlinearity and makes the linear interference effect permanent.

\subsubsection{Schrodinger, Heisenberg, and Dirac's Formulation of Non- Relativistic Quantum Mechanics. Nature of the Stationary States}

Schrodinger's wave equation describes the continuous evolution for a system not already on one of the energy stationary states. Heisenberg's matrix treatment, on the other hand, deals directly with transition probabilities between the discreet stationary states. The two approaches are complementary, and their formal equivalence can be demonstrated (Schrodinger 1926b) especially through the transformation theory developed by Dirac $(1926,1927,1958)$.

However, one can demonstrate that something is missing from these formulations through a simple example, that of the trace of a single particle in a Wilson cloud chamber (Mott 1929). Since Schrodinger's picture predicts the dispersion of the particle wavepacket during propagation, and Heisenberg's picture describes the discontinuous jumps between stationary state, neither can account for the continuous and yet sharply-defined trace of a particle in the cloud chamber. In the new ontology, however, this continuous and thin trace is interpreted as produced by a series of environmentally induced phase transitions - i.e. the interaction of the particle wavepacket with the cloud chamber gaseous background. The thin trace is a dynamical balance of the dispersion tendency of the single particle wavepacket during propagation, and the wavefunction collapse tendency onto the successive positional eigenstates.

A quantum operator in general probes global instead of local characteristics. The operators that commute have the same eigenfunction set. The eigenvalues and eigenfunctions of operators are related through:

$$
A \Psi_{A, n}=a_{n} \Psi_{A, n}
$$

The underlying meaning of this operation in the new ontology is that certain 
operators preserve the modal structure of the corresponding modes. If an operator operates on a state that is not one of its eigenstates, it induces a phase transition and causes the original state to collapse onto one of its eigenstates.

It is interesting to note that even though the eigenvalue of a stationary state is a constant (call it $\alpha$, the eigenfunction is in general time dependent (the particular form of the eigenfunction, if it is a simultaneous eigenstate of

the Hamiltonian as well, is $\mid \alpha>\exp \left(\frac{-i E_{\alpha} t}{\hbar}\right)$ (see, e.g., Sakurai 1985). Therefore quantum mechanical stationary state is a kind of dynamical equilibrium in constant evolution, consistent with the nonequilibrium stationary state picture (Zhang 1998) we have proposed.

In practice, apart from stationary states and freely-evolving wavefunctions, the density matrix formalism (Von Neumann 1927) has also been employed to describe quantum systems that are thought to be statistical mixtures or ensembles. Since we adopt a realist ontology, no physical systems will actually be in a statistical mixture state. The physical universe is described as a series of hierarchies defined by successive phase transitions, which form quasi-stationary structures. The apparent success of the density matrix approach is understood as the intrinsic harmonic nature of the evolution of the parts and parcels of these quasi-stationary states, and the effect of time average in this case mimics the effect of ensemble average.

\subsubsection{Extent of a Quantum Measurement Process}

A quantum measurement is in general a non-local process, and in the new ontology its result is not determined soly by the localized measuring instrument and the object being measured, as manifested by the probabilistic nature of the measurement results. Quantum measurements also involve the "give and take" with the rest of the universe, as evidenced in the previously-mentioned position/momentum pair of measurements. This "give and take" with the rest of the universe accounts for the apparent violation of energy conservation in many quantum measurement processes. It also provides a natural explanation to the paradoxical fact that the accelerated electrons radiate in 
certain cases (as when they travel freely in straight lines) and not in other cases (as when they are in the Bohr atoms).

A pure quantum mechanical resonance remains a modal resonance spreading out in space until the moment of detection. Detection shows quantum behavior because the "remainder" of the stuff in the universe cavity shows quantum behavior. Therefore during the emission or detection of a "particle" there has to be a "give and take" with the background field (or the rest of the universe) to make up the difference. The detected particle is no longer the same particle during propagation because of the exchange with the universal background.

The strongest support for the involvement of the universe resonant cavity during the quantum measurement process is actually the constancy of the elementary particle characteristics among many different physical processes. Without a global resonant cavity to determine the modal characteristics, we would not have such things as elementary particles or for that matter fundamental constants themselves. The identity of particles is the result of their being the same mode. Other examples of the extended nature of quantum interactions are phonons and plasmon-mediated processes in solid state physics.

The global nature of the interaction/phase transition is also reflected in the "photon scattering" experiment of the atom interferometer (Schmiedmayer et al. 1997). During those single photon scattering events the phase imprints on the two arms of the atom interferometer reflected the phase difference of the photon wavefunction where it intersects the two arms of the interferometer, even though scattering of a single photon off a single atom wave is supposedly a single event. This is no longer so if the two arm's separation is large enough - in that case the lost coherence will not be able to be recovered.

\subsubsection{Wave-Particle Duality}

The wave-particle duality is one of the puzzling characteristics of the quan-

tum world, which contrasts sharply to the behaviors we are used to in the 
classical world. The wave-particle duality is partly what Bohr based his Complementarity Principle upon (Bohr 1928). Modern quantum optical experiments show that a free-propagating beam of photons show simultaneously the wave and the particle characteristics (Aichele et al. 2004).

The wave-particle duality is manifest most clearly in the de Broglie relation $(p=h / \lambda)$ and Planck relation $(E=h \nu)$, each on one side indicates a pure wave characteristic $(\nu$ and $\lambda)$ and on the other a pure particle characteristic $(E$ and $p)$. The seeming contradictory characteristic is easily clarified in the new ontology. Here a particle is more of a pure resonance when it is a wave mode and is spread out. When it is a localized particle, it is in a mixed resonant state, or the superposition of pure states.

In fact, in the quantum field theories, only the fields are localized, but field quanta are spatially extended. These spatially distributed field quanta arrive from the first approximation of the solution of field equations in the non-interacting limit, which simplifies analysis and is the source of the name "particle".

\subsubsection{Uncertainty Principle, Quantum Commutation Relation, and Classical Poisson Bracket}

Heisenberg (1926) wrote in a letter to Pauli explaining the implications of the position-momentum commutation relation and the associated uncertainty principle: "The equation $p q-q p=h / 2 \pi i$ thus corresponds always in the wave representation to the fact that it is impossible to speak of a monochromatic wave at a fixed point in time (or in a very short time interval) ... Analogously, it is impossible to talk of the position of a particle of fixed velocity". Schrodinger (1930) subsequently proved the general relation between the commutation relations and the uncertainty relations. The founders of quantum mechanics thus were well aware of the connection between the two kinds of relations. A general derivation of the uncertainty relations from the commutator relations can be found in Sakurai (1985, p.34).

The uncertainty principle is thus just another way of writing the commutation relations, which themselves are deterministic. The uncertainty prin- 
ciple itself seemed to have later acquired more prominence in the quantum discussions only because of its intimate relation to the probabilistic outcome of quantum measurements.

It is well-known that quantum commutation relations can be linked to classical Poisson brackets in the "correspondence principle" sense of the quantum to classical transition (Goldstein 1980, p.399). This tight correlation between the two formulations demonstrates once again that the physics in the two regimes is closely linked, and the form of classical dynamics itself is already a result of the modal selection in the universe resonant cavity (we will comment more on this in section 3.2.10).

The special characteristic of the quantum commutation relations of course is the ever-present Planck's constant $\mathrm{h}$, which sets the scale of quantum interactions. The historical introduction of Planck's constant of course is through the well-known exploration of the functional dependence of blackbody radiation on wavelength and temperature (see Agassi 1993 for a chronological account), which leads to Planck's discovery of the famous $E=h \nu$ equation signaling the beginning of the quantum era. Once the value of $h$ is obtained by a comparison of Planck's blackbody formula with the empirical blackbody curve, its subsequent entry into quantum mechanics is through a series of "correspondence" type of analogies: i.e. Bohr's assumption of discrete energy levels of atoms as the origin of discrete spectra; Heisenberg's ingenious application of correspondence principle which leads to the first incidence of a quantum commutation relation; de Broglie's generalization of the quanta energy and momentum relations to that of material particles; and Schrodinger's derivation of his famous wave equation through the integration of de Broglie formula and classical mechanics. Thus, the root of quantized behavior (or at least our discovery of it) goes back to the source of cosmic blackbody radiation (even though at the time of Planck he was mostly focused on the blackbody radiation in a small box), which we know is related to the universe resonant cavity as a whole!

The relation between the quantum and classical modal behavior allows us to borrow the tools developed for analyzing classical nonequilibrium structures (Zhang 1996, 1998) and resonant phenomena (Cohen 1995) towards a 
deeper understanding of the behavior of quantum systems.

\subsubsection{Quantum Vacuum}

After quantizing space with a set of modes using the commutation or anticommutation relations, we expect to end up with some leftovers, and these leftover fractional modal content we propose is the composition of vacuum fluctuations.

The vacuum field fluctuates because the resonant components keep evolving in the nonequilibrium universe cavity, just like in another example of such a nonequilibrium phase transition, that of the spiral structure in galaxies (Zhang 1998), where the individual star's trajectory keeps evolving and moving in and out of the spiral pattern even though total energy is conserved and the spiral pattern is meta-stable.

This picture provides a possible explanation of why many fundamental physical effects (such as Lamb shifts, Casimir effects, spontaneous emission, van der Waals forces, and the fundamental linewidth of a laser) can be explained equally successfully by adopting either the vacuum-fluctuation point of view or the source-field point of view (see, e.g., Milonni 1994 and the references therein).

Addition of metal plates as in Casimir effect changes the boundary condition of the entire vacuum, and force is thus needed to put the plates in. The energetically most favorable arrangement of the resonances is thus changed. So is the modal structure.

The relation of field quantization and vacuum fluctuation may also be related to the "fluctuation-dissipation theorem". The dissipative leaks into the vacuum is needed to maintain the stability of the nonequilibrium modes. The universe also has to be constantly evolving in order for the fundamental resonances to be stable. So the un-saturatedness and the constant evolving nature of the universe maybe a prerequisite for setting up the fundamental laws as we observe today. 


\subsubsection{Lifetime of Levels and Virtual Particles}

Since quantizations of physical systems do not happen in a closed box, but rather in an open and evolving universe, there is a continuous exchange of any given mode with the underlying continuum. The modal characteristics thus formed are not always sharp, which allowed the existence of wavepackets, as well as the finite lifetimes of "fundamental particles" and "eigenstates". These quasi-stationary states are generally in dynamical equilibrium. The quantization characteristic is manifestly the sharpest during the emission/detection process, which is a true non-equilibrium phase transition - but even there the emitted photons/particles may not immediately be in a momentum eigenstate. A photon during propagation is not always in a pure momentum eigenstate, but has smear, as we will comment further below.

Virtual particles are those which appear in a quantum electrodynamic calculation and do not satisfy energy and angular momentum conservation: They are "not on the mass shell" and are represented by the internal lines in Feynman diagrams (Feynman 1949; Dyson 1949). Their existence is another indication that a quantum phase transition involves the rest of the universe to "close the loop", and the conservation relation is restored for resonant interactions only when the phase transition is complete.

\subsubsection{First and Higher Order Coherence of Photons and Atoms. Identical Particles. Blackbody Radiation}

When Dirac commented that "A photon interferes only with itself" (Dirac 1958), he referred to the first order coherence properties of photons. Subsequent intensity interferometry experiments (Hanbury Brown \& Twiss 1954, 1956; Hanbury Brown 1974) had revealed that photons do interfere with one another, which are the higher order coherence properties of photons. Such first and higher order coherence properties were also confirmed for atoms in the atom interferometry experiments (see the contributions in Berman eds. 1997).

In the current ontology, the first order coherence of the atoms and pho- 
tons reveals their underlying wave and modal nature, whereas the higher order correlation is a manifestation of the finite- $Q$ nature of the universe resonant cavity, resulting in "non-pure" spatial modes which entangles the individual particles or field resonances. Due to the entanglement (as reflected in the Bose-Einstein or Fermi-Dirac statistics, for example, which can be regarded as different types of global mode organizations), after quantized emission a photon has a tendency to merge back to the universal "soup" of the background photon flux during propagation, unless the photon flux is so low that it can be described as spatially and temperally separated monophotonic states (Hong, Ou, \& Mandel 1987), in which case its degree of second order coherence $g^{2}(0)<1$ as is appropriate for photons in the non-classical photon number states (Loudon 1983). The analytical expressions for the degree of second order coherence for bosons and fermions show different expressions according to their respective wavefunction symmetries (Scully \& Zubairy 1997, p.125), and these statistics are only meaningful when the particle flux is high enough.

The existence of second or higher order coherence does not require that the wave be non-classical (i.e. quantized, see Hanbury Brown 1974). In some sense, a classical wave itself embodies the correlation between its constituent parts, and there is no clear dividing line between the classical and the quantum worlds. It is only by convention (or chronology) that we refer to the particle nature of the photons as nonclassical and the wave nature as classical, whereas for massive particles our convention is the reverse.

The correlation and entanglement characteristic is needed to make sense of the coherent length and coherent time of photon waves. It would be meaningless to talk in such terms if each photon is completely independent of the other ones. For coherent length to matter for the easiness of wavefunction collapse during a quantum measurement, as in the comparison of beam splitter decoherence in either the laser or Bose-Einstein condensate cases (Zhang 2005), the relevant particles must have mutual interactions during propagation. Otherwise only the first order coherence will matter. Furthermore, the correlation between the coherence length or coherence time with the bandwidth of electromagnetic radiation also indicates the global nature of the underlying modes, that the universe cavity is connected and shared among 
the different energy quanta.

The internal correlation is also revealed in the equation of blackbody radiation. Ehrenfest (1911) had already realized that independent quanta could only lead to Wien's law, but not Planck's law. Thus, if light quanta were to be described by the Planck distribution, they had to lack statistical independence and show wave-type correlation through satisfying Bose-Einstein statistics (Ehrenfest \& Kamerling-Onnes 1915; Bose 1924).

\subsubsection{Spontaneous Quantum Measurements in Naturally Occur- ing Orders}

Under the new ontology there is no longer a dichotomy between the classical and the quantum world. The classical systems consist of subunits where "wavefunction collapse" have already been induced by nature, through naturally occurring boundary conditions. A macroscopic object does not possess an overall quantum mechanical wavefunction that freely evolves. The different hierarchies of the macroscopic system established by non-equilibrium phase transitions have quasi-autonomy.

The spontaneous nature of the phase transitions in natural systems helps to resolve "Schrodinger's Cat" type of paradoxes (Schrodinger 1935), since a naturally occurring "quantum measurement" does not have to involve a conscious observer. The cat in question was already in a definitive live or dead state before the observer opened the box, and not in a linear superposition state of the kind $a \cdot$ Alive $+b \cdot$ Dead. The possibility that naturally occurring orders are results of non-equilibrium phase transitions also explains the stability and reproducibility of these natural orders, i.e., the result of the non-equilibrium phase transitions is insensitive to the details of the initial-boundary conditions, and depends only on the gross nature of these conditions.

Such views have also been expressed by the founder of the dissipative structure theory I. Prigogine (1997): "But once it is shown that instability breaks time symmetry, the observer is no longer essential. In solving the time paradox, we also solved the quantum paradox". 


\subsubsection{The Form and Origin of Physical Laws. Values of Funda- mental Constants}

The physical laws, both classical and quantum, can often be derived from least action or variational principles (Goldstein 1980; Weinberg 1996). This behavior generally reflects the fact that the energy content of the process under concern is distributed globally, and the process samples the environmental/boundary conditions of the entire space of relevance. In the case of the classical trajectory of particles, this is reflected in Feynman's rule of sum over all possible paths (Feynman \& Hibbs 1965). The quantum laws of count statistics for identical particles (from which the Planck's law was derived from) are valid also because the universe is a connected resonant cavity.

The relations between symmetry and the conservation (Noether's theorem, see, e.g. Goldstein 1980) indicate that the dynamical equations (both classical and quantum) have already incorporated (or is consistent with) the symmetry of spacetime, i.e., the equations and laws themselves are selected by the universe resonant cavity. This is reasonable since both the equations and the fundamental constants are likely to be a result of spontaneous breaking of gauge symmetry, whose occurrence is closely related to the state of the matter in the universe.

If values of the fundamental constants are determined by the characteristics of the universe resonant cavity, the variation of the values of these "constants" with time (such as the recent observation of the likelihood of the variation of the fine-structure constant) would be naturally expected if the universe resonant cavity changes with time, e.g. due to the expansion of the universe and the associated evolution of matter distribution.

\subsection{Connections with the Past and Current Practices in Quantum Theories}

The discussions in the following had benefited greatly from the survey studies of the development of 20th century field theories of Cao (1997). 


\subsubsection{S-Matrix Theory}

In the 1950s and 60s the S-matrix theory, first proposed by Heisenberg (1943a,b, 1944) and later espoused by Chew and collaborators (Chew 1961 and the references therein), was enjoying the popularity the quantum field theories later enjoyed. The motivation for the theory is from processes such as Compton scattering which shows that light quanta comes in and goes off as approximate plane waves.

In this theory, the dynamics were not specified by a detailed model of interaction in spacetime, but were determined by the singularity structure of the scattering amplitudes, subject to the requirement of maximal analyticity. The success of the S-matrix approach is likely to be due to the fact that the underlying physics obeys global, modal characteristics. The calculation scheme in this theory was carried out on the mass shell, which is fine since it only concerns the input and output states, or the results of phase transition, and hence involves only asymptotic states where particles were outside each other's region of interaction.

\subsubsection{Quantum Field Theories: Renormalization and Feynman's Diagrammatic Approach}

In quantum field theories, such as quantum electrodynamics (QED), the interaction is transmitted by the discrete quanta of the electromagnetic field, and the interacting particles are considered the quanta of a fermion field. All such quanta can be created and destroyed.

Quantum field theory describes local interactions between particles and fields. In view of our previous discussions, that all interactions in the quantum world should be global by nature since they are in essence phase transitions in the universe cavity, we wonder how this could be reconciled with the apparent success of quantum field theory. In the following, we argue that many of the approaches adopted in quantum field theory calculations have in fact incorporated the global nature of the underlying processes.

For many quantum field processes such as scattering, the amplitudes are 
calculated from the chosen input and output states, which are generally expressed in plane wave forms. Invariably in these calculations the exact behavior of particles at the supposed "locations" of interaction are not specified in detail. This shows that the supposedly local quantum field calculations incorporated features of a global approach as in the S-matrix approach (Chew 1961). Scattering effectively becomes persistent scattering and neither starts nor stops.

Some of the common practices in quantum field calculations, such as Feynman's diagrammatic approach (Feynman 1949; Dyson 1949, which has its lineage in the S-matrix approach), are also integral representations of the entire phase transition process, described in terms of input and output states and omitting any detailed description of the "on-location" behavior of the interaction and particle generation/annihilation.

Another feature that indicates the insufficiency of a purely local description is the need of renormalization in quantum field theories. It was first realized by Weisskopf (1939) that the electromagnetic behavior of the electron in quantum field theory is not completely point-like, but is extended over a finite region. This extension, keeps the divergence of the self-energy within the logarithmic divergence which made the renormalization procedure possible.

The elementary phenomena in which divergences occur are the polarization of the vacuum and the self-energy of the electron (Schwinger 1948, 1949a,b). Each of these phenomena describes the interaction of one field with the vacuum fluctuations of the other field - once again a reflection of the global nature of quantum interactions. The overall effect is to alter the values of the electron charge e and mass $\mathrm{m}$, which are compensated by charge and mass renormalization.

The local interactions described by quantum field theories are for parts and parcels of fields. Yet like the case of any dissipative structures, the local characteristics are determined by the global organization and the global dynamical equilibrium. The local organization on the other hand reflects features of the global organization, in this case through the need of renormalization to obtain a self-consistent theory. 
The need for renormalization of any quantum field theories results not only from the local/global dichotomy between quantum field theories and quantum process, it also originate from the fundamental incompatibility of quantum theory with relativity theory. QED as a relativistic generalization of quantum mechanics is not self-consistent (other than in its non-interacting limit: in which case we obtain pure modes and no non-equilibrium phase transitions). The effect of spontaneous quantum measurements to order the processes into hierarchies is incorporated through the procedure of renormal-

ization. Empirical input is needed to construct a self-consistent theory due to the singular nature of phase transitions whose details cannot be models in a top-down deductive type of analysis. As Gross (1985) puts it: "Renormalization is an expression of the variation of the structure of physical interactions with changes in the scale of the phenomena being probed".

\subsubsection{Gauge Field Theories, Spontaneous Symmetry Breaking, and Effective Field Theories}

Gauge field theories emerged within the framework of quantum field theories, and is very powerful in exploring global features of field theories. In this theory the phase of a wave function becomes a new local variable. The invariance of a theory under the global phase change, or a gauge transformation of the first kind, entails charge conservation. The local gauge invariance is related to the electromagnetic interaction.

The existence of gauge principle for the determination of the forms of fundamental interactions indicates that the dynamical laws have been coselected in the universe resonant cavity as the processes themselves. The forms of the fundamental interactions must preserve and alter the modal structure of the underlying matter and energy distribution in a globally selfconsistent way. In this sense, the experience gained in obtaining the globally self-consistent solutions for classical nonequilibrium structures (Zhang 1998) can be used to gain deeper insight into the organization structure of quantum laws and fields.

Spontaneous symmetry breaking was developed as a mechanism to preserve gauge invariance when dealing with massive gauge quanta. The gener- 
ation of fundamental interactions through spontaneous symmetry breaking is viable since in general "The solutions of the equations will possess a lower symmetry than the equations themselves" (Landau 1958, quoted in Brown and Rechenberg 1988). The spontaneous symmetry breaking first proposed by Heisenberg and collaborators (Durr et al. 1959) is concerned with the low-energy behavior of the solutions and asserts that some low energy solutions exhibit less symmetry than the symmetry exhibited by the Lagrangian of the system, while others possess the full symmetry of the system.

Since the late 1970 s theorists gradually realized that the high-energy effects in gauge theories can be calculated without taking the cutoff in the renormalization approach to infinity. The cutoffs in these Effective Field Theories (Weinberg 1980) acquire physical significance as the embodiment of the hierarchical structure of quantum field theory, and as a boundary separating energy regions which are separately describable by different sets of parameters and different interactions with different symmetries. Non-renormalizable theories can also be treated as effective field theories incorporating empirical input from the experiments.

Thus it appears the new picture offered by the modern development of field theories points also to a kind of hierarchical organization of the highenergy world through successive phase transitions. Each layer of the hierarchy is quasi-autonomous, each has its own ontology and the associated "fundamental laws". The ontology and dynamics of each layer are quasi-stable, nearly (but not totally) immune to whatever happen in other layers.

\subsection{Issues Requiring Further Study}

\subsubsection{Connection of Quantum Mechanical Measurement Process with Relativity Theories}

The quantum measurement processes, especially the Einstein-Podolsky-Rosen (1935) type or delayed-choice (Wheeler 1978) type of experiments, clearly show both the global extent of the wavefunction and the superluminal nature of the wavefunction collapse. 
Since special relativity dictates that physical signals travel with a speed less than the speed of light $\mathrm{c}$, this element of the wavefunction collapse requires further study. Furthermore, we could ask questions such as whether the speed of a single-particle wavefunction collapse depends on the spatial spread of the wavefunction, and whether the instantaneous wavefunction collapse corresponds to instantaneous energy propagation as well.

In fact, the ontology of relativity theories may itself need to be further elucidated. Questions we could ask include: Why is there a speed-of-light limit? Is it only there for certain levels of hierarchy and not for others (see next section)? If in the eye of light, the world is but a single point, how could changes and evolution ever be possible? Does that mean for light the external degrees-of-freedom get transformed into the internal degreesof-freedom to allow evolution? The essential incompatibility of relativity theory and quantum mechanics, i.e. the loss of Lorentz invariance in quantum measurement processes needs to be further explored.

\subsubsection{Hierarchy of Laws and Mach's Principle}

We have commented that it appears that the speed of light limit holds for classical physical processes as well as non-measurement or non-phasetransition type quantum processes. This is an indication that the workings of the physical world may be organized into hierarchies. Even though in the lower hierarchy the speed of light is a fundamental limit to signal propagation, the same is not true for wavefunction collapse.

Furthermore, we expect that the establishment of the fundamental laws and the selection of fundamental constants through the resonant interaction in the universe cavity according to Mach's principle are also themselves processes which are not constrained by the speed of light limit: For otherwise the mere size of the universe and the cycle of propagations needed to resonantly establish a fundamental constant would make it impossible for these constants and laws to be universal and atemporal (at least over short time span). The statistical laws of physics, such as Einstein's A and B coefficients for spontaneous and stimulated emission (Einstein 1917), and Planck's radia- 
tion law, would not be valid and universal if the impact of the entire content of the universe is not communicated instantaneously. The variational approach for the selection of laws (as indicated by the Lagrangian type of derivation) only works if all possible paths are "explored" acausally. The fact that in the variational approach the different path explored are not constrained by the speed of light limit is evidenced from Feynman's path integral formulation (Feynman \& Hibbs 1965).

As commented by Barbour \& Pfister (1995), "It is often not sufficiently appreciated how kind nature has been in supplying us with 'subsystems' of the universe which possess characteristic properties that can be described and measured almost without recourse to the rest of the universe. ... On the other hand, it is evident that basic concepts such as 'inertia' and 'centrifugal force' cannot be understood and explained within the context of the subsystems themselves, but at best by taking into account the rest of the universe". That refers to Mach's original speculations on the origin of inertia. By the same token other properties of the universe cavity such as the quantization unit $\mathrm{h}$ are expected to be determined by the entire universe as well.

Questions requiring further work to answer include: What characteristics of the universe resonant cavity generate the fundamental constants (presumably as eigenvalues)? How do the different levels of hierarchy interact? etc.

\subsubsection{Causality of the Quantum Processes}

The global nature of the quantum phase transitions also demands us to alter our traditional notions of cause and effect. The boundary between causes and effects are now blurred, and there is now only an intertwined co-evolution of all the parts of the universe resonant cavity, strictly speaking - though the hierarchical structure of orders in the universe due to the successive phase transitions allows the emergence of macroscopic objects and classical phenomena which conform to our notion of the cause-and-effect.

At a deeper level though, we could not ignore the fact that we have now a kind of more distributed and global causality. It was already known since the early days of quantum mechanics that radiative phenomena cannot be 
analyzed causally. The stability of the atom shows that there cannot be a classical type of causal process in the radiability of the atom. Scattering of quantum particles likewise is also a distributed process which could not be analyzed as a causal sequence. In this picture, forces as exchange of particles serve to realize an energetically more favorable configuration.

Other evidence of the new causality include the description of many natural processes using analytical signals. To know an analytical signal at time t we have to know the real signal at all times: This once again shows the global influence of the modal behavior.

Mach himself summarizes this intermingled state of cause and effect in the self-sustained universe as follows: "Thus the law of causality is sufficiently characterized by saying that it is the presupposition of the mutual dependence of phenomena. Certain idle questions, for example, whether the cause precedes or is simultaneous with the effect, then vanish by themselves" (Mach 1911); as well as "The universe is not given us twice, but only once".

The many consequences of this new meaning of quantum causality need to be further explored.

\subsubsection{The Possibility of Free Will in A Universe Governed by Deterministic Physical Laws}

Does the deterministic ontology presented in this paper prohibit the emergence of free-will of sentient beings, which we know from personal experiences do exist?

From the work of Yang and Lee (1952), we know that the sharpness of the phase transitions in classical statistical systems is related to the effective number of degree-of-freedom of the system. In the new ontology, the effective degree-of-freedom of the physical phenomena in the universe resonant cavity is non-denumerably infinite, since we are dealing with a true continuum made up of the smooth reservoir of energy, and the discreteness we observe in the physical universe is merely resonant modal behavior formed in the universe cavity. 
The infinitely-sensitive dependence on the details of perturbations of an infinite degree-of-freedom system is likely to be the cause of the indeterministic factor in the generation of free will, though the detailed workings of the free-will generation process still require further study. This indeterministic factor in free will is not to be confused with the quantum uncertainty relations, which are themselves only a derived property of the exact quantum commutation relations. The indeterministic factor in the phase transitions of an infinite degree-of-freedom system allows chance to play a role, and introduces an element of history into the course of evolution of the universe.

\section{Conclusions}

In this paper, we argue that even though the quantum mechanical paradigm is the correct description of microscopic phenomena, unitary evolution is not the only guiding principle. To that we need to add the principle of phasetransition in the universe resonant cavity, and this principle is responsible for organizing both the physical laws and natural orders into hierarchies. Both the amplitude and the phase of the quantum mechanical wavefunction are substantial in nature, and the probability element is removed from the foundations of quantum mechanics. An effectively classical ontology is provided for the quantum processes.

The new ontology on the foundations of quantum mechanics and especially on the nature of quantum measurements leads to a coherent interpretation of a wide spectrum of phenomena in the quantum as well as the classical world. It also provides a framework for understanding and coherently linking many diverse results in quantum field theories which have been accumulated over the past few decades. As a working hypothesis, it could serve to stimulate a new synthesis on our understanding of the workings of the physical universe.

This research was supported in part by funding from the Office of Naval Research. 


\section{References}

Agassi, J. 1993, Radiation Theory and the Quantum Revolution (iBasel: Birkhauser)

Aharonov, Y., \& Bohm, D. 1959, Phys. Rev. (Ser. 2) 115, 485

Aichele, T., Herzog, U., Scholz, M., \& Benson, O. 2004, quant-ph/0410112

Alley, C.O., Jakubowicz, O.G., \& Wicks, W.C. 1987, in Proc. Second International Symposium Foundations of Quantum Mechanics, eds. M. Namiki et al. (Tokyo: Phy. Soc. Japan), p.36

Aspect, A. 1976, Phys. Rev. D14, 1944

Aspect, A., Grangier, P., \& Roger, G. 1981, Phys. Rev. Lett. 47, 460

Barbour, J. \& Pfister, H. 1995, Mach's Principle: From Newton's Bucket to Quantum Gravity (Boston: Birkhauser)

Bell, J.S. 1965, Physics, 1, 195

Berman, P.R. ed. 1997, Atom Interferometry (San Diego: Academic Press)

Bohr, N. 1928, Nature, 121, 580

Born, M. 1926, Zeitschrift fur Physik, 37, 863, English version in Wheeler \& Zurek eds. Quantum Theory and Measurement (1983), p. 52

Bose, S.N. 1924, Zeitschrift fur Physik, 26, 178

Braginsky, V.B., \& Khalili, F.Y. 1992, Quantum Measurement, (Cambridge: Cambridge Univ. Press)

Briegel, H.J., Englert, B.G., Scully, M.O., \& Walther, H. 1997, in Atom Interferometry, ed. P.R. Berman (San Diego: Academic Press), p. 217

Brown, L.M., \& Rechenberg, H. 1988, in Landau's work on quantum Field theory and high energy physics (1930-1961), Max Planck Institute preprint, 
MPI-PAE/Pth 44/88 (July 1988)

Cao, T.Y. 1997, Conceptual Developments of 20th Century Field Theories (Cambridge: Cambridge Univ. Press)

Chew, G.F. 1961, S-Matrix Theory of Strong Interactions (New York: Benjamin)

Chiao, R.Y., Kwait, P.G., \& Steinberg, A.M. 1994, in "Advances in Atomic, Molecular, and Optical Physics", B. Bederson \& H. Walther eds., vol 34, (New York: Academic Press), p. 35

Cohen, L. 1995, Time-Frequency Analysis (New Jersey: Prentice Hall)

DeWitt, B.S. 1970, Phys. Today, 23, 30

DeWitt, B.S. 2005, Physics Today, January issue

Dirac, P.A.M. 1926, Proc. Royal Soc. London, vol. 112, No. 762, p. 661

Dirac, P.A.M. 1927, Proc. Royal Soc. London, vol. A113, p. 621

Dirac, P.A.M. 1958, The Principles of Quantum Mechanics, 4th edition (Oxford: Oxford Univ. Press)

Durr, H.P., Heisenberg, W., Mitter, H., Schlieder, S., \& Yamazaki, K. 1959, Zeitschrift fur Naturforschung, 14A, 441

Dyson, F. 1949, Phys. Rev. 75, 486

Ehrenfest, P. 1911, Ann. Phys., 36, 91

Ehrenfest, P. \& Kamerling-Onnes, H. 1915, Proc. Amsterdam Acad. 23, 789

Einstein, A. 1917, Phys. Z. 18, 121, English translation by D. ter Harr 1967, The Old Quantum Theory (New York: Pergamon Press), p.167

Einstein, A., Podolsky, B., \& Rosen, N. 1925, Phys. Rev. 47, 777

Everett III, H. 1957, Rev. Modern. Phys. 29, 455 
Feynman, R.P. 1949, Phys. Rev. 76, 769

Feynman, R.P., \& Hibbs, A.R. 1965, Quantum Mechanics and Path Integrals (New York: McGraw-Hill)

Glansdorf, P., \& Prigogine, I. 1971, Thermodynamic Theory of Structure, Stability and Fluctuations (New York: Wiley-Interscience)

Goldstein, H. 1980, Classical Mechanics, 2nd ed. (Menlo Park: AddisonWesley)

Gross, D. 1985, in Recent Developments in Quantum Field Theories, J. Ambjorn, B.J. Durhuus, and J.L. Petersen, eds., (Amsterdam: Elsevier), p. 151

Hanbury Brown, R. 1974, The Intensity Interferometer (London: Taylor and Francis)

Hanbury Brown, R. \& Twiss, R.Q. 1954, Philos. Mag., Ser. 7, No. 45, 663

Hanbury Brown, R. \& Twiss, R.Q. 1956, Nature, 178, 1046

Heisenberg, W. 1926, in Pauli, W., Wissenschaftlicher Briefwechsel mit Bohr, Einstein, Heisenberg, u. A., Band I: 1919-1929, eds. Hermann, A., Meyenn, K.V., and Weisskopf, V.E. (Berlin:Springer, 1979)

Heisenberg, W. 1927, Zeitschrift fur Physik, 43, 172, English version in Wheeler \& Zurek eds. Quantum Theory and Measurement (1983), p. 62

Heisenberg, W. 1943a, Zeitschrift fur Physik, 120, 513

Heisenberg, W. 1943b, Zeitschrift fur Physik, 120, 673

Heisenberg, W. 1944, Zeitschrift fur Physik, 123, 93

Hellmuth, T., Walther, H., Zajonc, A., \& Schkeich, W. 1987, Phys. Rev. A, 35,2532

Hong, C.K., Ou, A.Y., \& Mandel, L. 1987, Physical Review Letters, Vol. 59, No. 18,2044 
Koschmieder, E.L. 1993, Benard Cells and Taylor Vortices (Cambridge: Cambridge Univ. Press)

Landau, L.D., \& Peierls, R. 1931, Z. Phys. 69, 56

Loudon, R. 1983, The Quantum Theory of Light (Oxford: Oxford Univ. Press)

Mach, E. 1911, History and Root of the Principle of the Conservation of Energy, P. Jourdain, trans. Chicago: Open Court (Translation of Mach 1872), p.61

Milonni, P.W. 1994, The Quantum Vacuum: An Introduction to Quantum Electrodynamics (San Diego: Academic Press)

Moore, W.J. 1989, Schrodinger: Life and Thought (Cambridge: Cambridge Univ. Press)

Mott, N.F. 1929, Proc. Royal Soc. London, A126, 79

Nambu, Y. 1949, Prog. Theor. Phys. 4, 82

Namiki, M., Pascazio, S., \& Nakazayo, H. 1997, Decoherence and Quantum Measurements (New Jersey: World Scientific)

Nicolis, G., \& Prigogine, I. 1977, Self-Organization in Nonequilibrium Systems (New York: Wiley-Intersciences)

Popper, K.R. 1935, The Logic of Scientific Discovery (Vienna: Verlag von Julius Springer), first English edition published in 1959 by Hutchinson \& Co (London)

Prigogine, I. 1997, The End of Certainty (Freepress)

Sakurai, J.J. 1985, Modern Quantum Mechanics (New York: Addison-Wesley) Schmiedmayer, J., Chapman, M.S., Ekstrom, C.K., Hammond, T.D., Kokorowski, D.A., Lenet, A., Rubenstein, R.A., Smith, E.T., \& Pritchard, D.E. 1997, in Berman ed. Atom Interferometry (San Diego: Academic Press), p.2 
Schrodinger, E. 1926a, Die Naturwissenschaften 28, p.664

Schrodinger, E. 1926b, Annalen der Physik (4), vol. 79, p.45

Schrodinger, E. 1926c, Annalen der Physik (4), vol. 81, p.109

Schrodinger, E. 1930, S.B. Preuss. Akad. Wiss., p.296, see also W.T. Scott 1967, "Erwin Schrodinger: An Introduction to His Writings", (Amherst, Univ. Mass. Press), p.64

Schrodinger, E. 1935, Naturwissenschaften 23, 807; English translation by J.D. Trimmer, 1980, Proc. Am. Phil. Soc. 124, 323

Schwinger, J. 1948, Phys. Rev. 74, 1439

Schwinger, J. 1949a, Phys. Rev. 75, 651

Schwinger, J. 1949b, Phys. Rev. 76, 790

Scully, M.O., \& Zubairy, M.S. 1997, Quantum Optics (Cambridge: Cambridge University Press)

Von Newmann, J. 1927, Wahrscheinlichkeits-theoretischer Aufbau der Quantenmechanik, Gottinger Nachrichten I, No. 10, 245

Von Newmann, J. 1932, Mathematische Grundlagen der Quantem Mechanik. (Berlin: Spinger)

Weinberg, S. 1995, The Quantum Theory of Fields (Cambridge: Cambridge Univ. Press)

Weinberg, S. 1980, Phys. Lett. 91B, 51

Weisskopf, V.F. 1939, Phys. Rev. 56, 72

Wheeler, J.A. 1978, in Mathematical Foundations of Quantum Theory, A. R. Marlow ed. (New York: Academic Press), p.9

Wheeler, J.A., \& Zurek, W.H. 1983, Quantum Theory and Measurement (Princeton: Princeton Univ. Press) 
Wigner, E. 1963, Ameri. J. Phys., 31, 6

Yang, C.N. 1987, in Schrodinger: Centenary Celebration of a Polymath

Yang, C.N., \& Lee, T.D. 1952, Phys. Rev. 87, 404

Zee, A. 2003, Quantum Field Theory in a Nutshell (Princeton: Princeton Univ. Press)

Zhang, X. 1996, Astrophysical Journal, 457, 125

Zhang, X. 1998, Astrophysical Journal, 499, 98

Zhang, X. 1999, Astrophysical Journal, 518, 613

Zhang, X. 2003, invited review presented in the 2nd APCTP workshop on astrophysics, Formation and Interaction of Galaxies, ed. H.M. Lee, Journal of Korean Astronomical Society, 36, 223, 2003

Zhang, X. 2004, invited talk presented in the international conference on Penetrating Bars through Masks of Cosmic Dust: Hubble Tuning Fork Strikes a New Note, eds. D. Block et al., Astrophysics and Space Science, 319, 317

Zhang, X. 2005, Naval Research Lab memorandum report, NRL/MR/7210$05-8866$

Zurek, W.H. 2002, Los Alamos Science, No. 27, p.2 